\title{
Problem-orientated categorisation of "other conditions" seen in a genitourinary medicine clinic
}

\author{
R S PATTMAN AND C B S SCHOFIELD \\ From Ward 34, Newcastle General Hospital, Newcastle upon Tyne
}

SUMMARY The case sheets of patients coded as D2 (other conditions requiring treatment) and D3 (other conditions not requiring treatment) in 1981 were reclassified on a problem-orientated basis. Ten discreet categories were delineated with ease. Women with non-specific vaginitis and men with Gardnerella vaginalis urogenital infection, usually coded D2 or C4, should be reclassified under the C group of conditions as, for example, "Gardnerella or other bacterial genital infections."

It is suggested that codes D2 and D3 be replaced by the following nine categories: genital skin lesions or rashes; genitourinary symptoms; enteric conditions; other sexually transmitted infections; normal genital architecture, congenital conditions, or unconfirmed genital discharges; asymptomatic patients attending for routine examination; uninfected contacts of patients with sexually transmitted diseases; psychosexual problems; and others.

\section{Introduction}

In a recent article ${ }^{1}$ examining the deficiencies in the current recording of statistics from genitourinary medicine clinics in the UK Professor Adler described the D2 (other conditions requiring treatment) and D3 (other conditions not requiring treatment) categories as vague and a potential "dumping" ground. About $35 \%$ of the total cases seen in clinics are diagnosed under these headings and little information regarding the nature of the patient's true condition can be obtained.

The decision on whether to diagnose a condition as D2 or D3 is often arbitrary. Some patients, for example, with balanitis, will receive treatment while others will not, depending on the severity of the inflammation, the nature of the patient, and the whim of the clinician. Does giving advice and reassurance to a worried patient without abnormality or infection constitute treatment in the form of psychotherapy? If so, all such patients should be diagnosed as $\mathrm{D} 2$.

To resolve this problem the case sheets of patients diagnosed as D2 and D3 in 1981 were examined retrospectively and further assigned on a problemorientated basis to one of 10 categories.

Address for reprints: Dr R S Pattman, Ward 34, Newcastle General Hospital, Westgate Road, Newcastle upon Tyne NE4 6BE

Accepted for publication 9 August 1982

\section{Results}

A total of 7727 cases were diagnosed in Newcastle during 1981, $990(12 \cdot 8 \%)$ being coded D2 and 2168 $(28 \cdot 1 \%)$ D3. The problem-orientated categories, incorporating both $\mathrm{D} 2$ and $\mathrm{D} 3$ conditions, are set out in the table. Twenty per cent of women had a nonspecific vaginitis (category 1), the vast majority associated with Gardnerella vaginalis.

TABLE Problem-orientated categories of patients coded D2 or D3. (Percentages are given in parentheses).

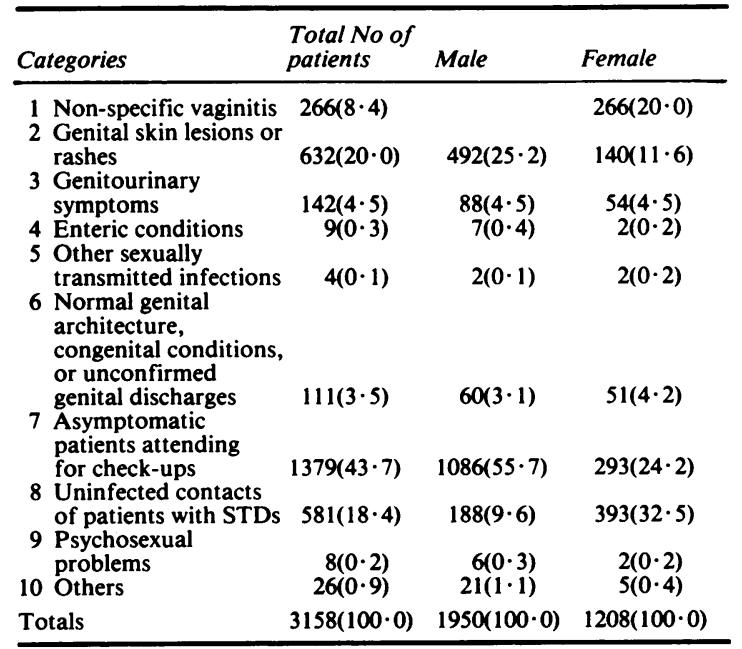


Twenty per cent of patients had genital skin lesions or rashes (category 2), the most common conditions being a non-specific balanoposthitis or vulvitis, but also included are such perigenital conditions as tinea cruris. The majority of patients with genitourinary symptoms (category 3) had a confirmed urinary tract infection or dysuria where sexually transmitted infection was excluded, while some women complained of irregular uterine bleeding or lower abdominal pains which resolved without the need for referral.

Enteric conditions (category 4) included patients with traumatic anal lesions as well as cases of giardiasis, amoebiasis, Shigella dysentery, and enterobiasis, as well as hepatitis B virus infections. Five out of seven of the latter cases were omitted as it was a coincidental finding, but all were referred to a gastroenterologist as part of a survey and were classified under D4 (other conditions referred elsewhere). Of the four patients with other sexually transmitted infections (category 5), two had meningococcal genital infections and two haemolytic streptococcal tonsillitis. Patients complaining of a urethral or vaginal discharge but lacking clinical or microbiological evidence of infection were combined with those who were worried about their normal genital architecture.

Asymptomatic patients attending for check-ups (category 6) formed the largest category $(43 \cdot 7 \%)$ and included most men $(55 \cdot 7 \%)$. Of this group, $13 \cdot 4 \%$ had already received treatment from their general practitioners and attended for a second opinion while a few $(1.0 \%)$ were babies for adoption.

Uninfected contacts of patients already attending (category 8 ) accounted for $18.4 \%$ of patients coded D2 or D3 and included the largest category among women $(32 \cdot 5 \%)$. Although there was no evidence of infection $26.7 \%$ did receive prophylactic treatment, mainly male contacts of women with trichomoniasis (C6) and female contacts of men with non-specific genital infections (C4).

Retrospectively only eight patients could be identified as having attended solely because of psychosexual problems (category 9). A small number of ill-defined conditions and causes were grouped together as "others" (category 10). These included patients with extragenital skin conditions such as pityriasis rosea and those patients who, after being registered, left the clinic before being examined or who refused to be examined.

\section{Discussion}

Most cases when reviewed retrospectively could be allocated to one or other of the problem-orientated categories as shown. The one misfit appeared to be non-specific vaginitis. This is a common condition with distinct clinical and microbiological features ${ }^{2}$ and

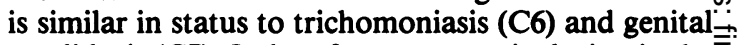
candidosis (C7). It therefore warrants inclusion in thes? $\mathrm{C}$ group of sexually transmitted infections as, say $\mathrm{C} 13$. As $G$ vaginalis is also associated with urogenitalo infection in men $^{3-5}$ the title of the category will need to $\frac{\overline{\bar{s}}}{\overline{7}}$ take this into account, so possibly "Gardnerella or $\stackrel{\mathbb{Q}}{\mathbb{Q}}$ other bacterial genital infection" might be moreo appropriate. Inclusion under non-specific genital ${ }^{\infty}$ infection (C4) has been proposed, ${ }^{16}$ but this would. seem to be inappropriate as it would tend towards $\overrightarrow{-}$ making $C 4$ another dumping ground. There is a need ${ }_{\sigma}$ rather to expand $\mathrm{C4}$ on an anatomical basis as has? already been done for gonorrhoea and in addition toir identify infections caused by specific organisms such. as Chlamydia trachomatis.

The nine other categories could then replace the $e_{\circ}^{\omega}$ current D2 and D3 codes. There does not appear to be any difficulty in identifying those patients to $\mathrm{be}_{\mathrm{T}}^{-}$ allocated to them. Of those with symptoms few of ours patients had enteric conditions, but in other clinics the numbers may be large. It is valuable to record cases of other infections which may be sexually transmitted such as infectious mononucleosis, cytomegalovirus $\oplus_{\infty}^{\circ}$ infection, oral herpes simplex virus infection inc contacts of patients with herpes genitalis $(\mathrm{Cl})$, ando urogenital Neisseria-type infections, other than Neisseria gonorrhoeae, with important medico-legal implications. Retrospectively several cases were missed which would have been included if the $\frac{2}{2}$ diagnosis was made at the time of attendance.

Patients with psychosexual problems usually have $\overrightarrow{\vec{\partial}}$ vague symptoms, and the number we were able to identify retrospectively was small. The number who also had a sexually transmitted disease has not been considered, and it is certain that a proportion of those? coming for a check-up, but who had not run a risk of $\frac{}{3}$ infection within three months of attending (194 men.and 30 women), did have some psychosexualo problems. All these could have been identified if a separate diagnostic category had been available.

Of those without symptoms it is important to be able to differentiate between those coming of their own accord and those attending because of contact tracing efforts. The former gives some indication of the standing of the clinic in the eyes of the local community while the latter does add somew information on the work load of the health advisers working in the clinics. Currently little information iso available in Part G of Form SBH60, only that of contact tracing efforts for those with syphilis and $\stackrel{?}{+}$ gonorrhoea. In Newcastle during 1981 this amounted 0 to only $32.0 \%$ of contacts attending and $23.6 \%$ of those requiring treatment. Full information is of course available in Scotland from Form ISD(D) $5, \stackrel{\mathbb{Q}}{\varrho}$ Table 3. 
We believe that categorising conditions currently coded D2 and D3 on a problem-orientated basis would provide a truer record of the conditions seen in genitourinary medicine clinics and enable the work load requirements to be gauged more accurately. It would also eliminate inter-clinic differences in coding, a diagnosis currently depending on the individual clinician's decision whether or not to give any treatment. A prospective pilot study, possibly organised by the British Co-operative Clinical Group in collaboration with the academic department of genitourinary medicine of the Middlesex Hospital, to assess the feasibility of such a system would be of value before it was submitted as an alternative to the current diagnostic coding.

\section{References}

1. Adler MW. Current routine statistics in the United Kingdom: room for improvement? Br J Vener Dis 1981;57:83-8.

2. Gardner $\mathrm{HL}$, Dukes CD. Haemophilus vaginalis vaginitis. Am J Obstet Gynecol 1955;69:962-76.

3. Kinghorn GR, Jones BM, Chowdhury FH, Geary I. Balanoposthitis associated with Gardnerella vaginalis infection in men. Br J Vener Dis 1982;58:127-9.

4. Abercrombie GF, Allen J, Maskell R. Corynebacterium vaginale urinary tract infection in a young man. Lancet $1978 ; \mathrm{i}: 766$.

5. Dawson SG, Ison CA, Easmon CSF. Vaginitis revisited. Br Med J 1981;ii:1333-4.

6. Belsey EM. Epidemiological treatment of gonorrhoea and nonspecific genital infection in female sexual contacts. Br J Vener Dis 1982;58:113-6. 